\title{
Some results on the partial orderings of block matrices
}

Xifu Liu* and Hu Yang

* Correspondence:

liuxifu211@hotmail.com

College of Mathematics and

Statistics, Chongqing University,

Chongqing 401331, China

\begin{abstract}
Some results relating to the block matrix partial orderings and the submatrix partial orderings are given. Special attention is paid to the star ordering of a sum of two matrices and the minus ordering of matrix product. Several equivalent conditions for the minus ordering are established.

Mathematics Subject Classification (2000): 15A45; $15 \mathrm{~A} 57$
\end{abstract}

Keywords: Matrix partial orderings, Moore-Penrose inverse, Block matrix

\section{Introduction}

Let $C^{m \times n}$ denote the set of all $m \times n$ matrices over the complex field $C$. The symbols $A^{*}, R(A), R^{\perp}(A), N(A)$ and $r(A)$ denote the conjugate transpose, the range, orthogonal complement space, the null space and the rank of a given matrix $A \in C^{m \times n}$.

Furthermore, $A^{\dagger}$ will stand for the Moore-Penrose inverse of $A$, i.e., the unique matrix satisfying the equations [1]:

$$
A X A=A X A X=X(A X)^{*}=A X(X A)^{*}=X A .
$$

Matrix partial orderings defined in $C^{m \times n}$ are considered in this paper. First of them is the star ordering introduced by Drazin [2], which is determined by

$$
A \stackrel{*}{\leq} B \Leftrightarrow A^{*} A=A^{*} B \text { and } A A^{*}=B A^{*},
$$

and can alternatively be specified as

$$
A \stackrel{*}{\leq} B \Leftrightarrow A^{\dagger} A=A^{\dagger} B \text { and } A A^{\dagger}=B A^{\dagger} .
$$

Modifying (1.2), Baksalary and Mitra [3] proposed the left-star and right-star orderings characterized as

$$
\begin{aligned}
& A * \leq B \Leftrightarrow A^{*} A=A^{*} B\left(\text { or } A^{\dagger} A=A^{\dagger} B\right) \text { and } R(A) \subseteq R(B), \\
& A \leq * B \Leftrightarrow A A^{*}=B A^{*}\left(\text { or } A A^{\dagger}=B A^{\dagger}\right) \text { and } R\left(A^{*}\right) \subseteq R\left(B^{*}\right) .
\end{aligned}
$$

The second partial ordering of interest is minus (rank subtractivity) ordering devised by Hartwig [4] and independently by Nambooripad [5]. It can be characterized as

$$
A \overline{\leq} B \Leftrightarrow r(B-A)=r(B)-r(A),
$$

(c) 2011 Liu and Yang; licensee Springer. This is an Open Access article distributed under the terms of the Creative Commons Attribution License (http://creativecommons.org/licenses/by/2.0), which permits unrestricted use, distribution, and reproduction in any medium, provided the original work is properly cited. 
or

$$
A \overline{\leq} B \Leftrightarrow A B^{\dagger} B=A, B B^{\dagger} A=A \text {, and } A B^{\dagger} A=A
$$

From (1.2), (1.4) and (1.5), it is seen that

$$
\begin{aligned}
& A \stackrel{*}{\leq} B \Leftrightarrow A^{*} \stackrel{*}{\leq} B^{*}, \\
& A * \leq B \Leftrightarrow A^{*} \leq * B^{*} .
\end{aligned}
$$

Hartwig and Styan [6] considered the rank subtractivity and Schur complement, and shown that

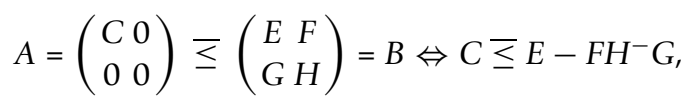

when the conditions $r\left(\begin{array}{c}F \\ H\end{array}\right)=r(H)=r(G H)$ are required, and $H^{-}$is a inner generalized inverse of $H$ (satisfying $H H^{\top} H=H$ ).

Recently, the relationships between orderings defined in (1.2)-(1.7) and their powers with the emphasis laid on indicating classes of matrices were considered by several authors [7-9]. The results on matrix partial orderings and reverse order law were considered by Benitez et al. [10]. In this paper, we focus our attention on the partial orderings of block matrices. Special attention is paid to the star ordering of a sum of two matrices and the minus ordering of matrix product. To our knowledge, there is no article yet discussing these partial orderings in the literature.

If $A \prec C, B \prec D$, an interesting question is that whether the partitioned matrices $(A B)\left(\right.$ or $\left.\left(\begin{array}{l}A \\ B\end{array}\right)\right)$ and $(C D)\left(\right.$ or $\left.\left(\begin{array}{l}C \\ D\end{array}\right)\right)$ have the same orderings, and the solutions will be given in the following sections. Also, the relations between $A \stackrel{*}{\leq} C, B \stackrel{*}{\leq} D$ and $A+B \stackrel{*}{\leq} C+D, A \leq B$ and $C A \overline{\leq} B$ are considered.

\section{Star partial ordering}

In this section, we give some results on the star partial orderings of block matrices.

Theorem 1 Let $A, C \in C^{m \times n}$ and $B, D \in C^{m \times k}$ be star-ordered as $A \stackrel{*}{\leq} C, B \stackrel{*}{\leq} D$. If $R$ $(A)=R(B)$, then $(A B) \stackrel{*}{\leq}(C D)$.

Proof. On account of (1.2) and (1.3), since $A \stackrel{*}{\leq} C, B \stackrel{*}{\leq} D$ and $R(A)=R(B)$, so

$$
\begin{aligned}
(A B)^{*}(A B) & =\left(\begin{array}{c}
A^{*} A A^{*} B \\
B^{*} A B^{*} B
\end{array}\right) \\
& =\left(\begin{array}{cc}
A^{*} C & A^{*} B B^{\dagger} D \\
B^{*} A A^{\dagger} C & B^{*} D
\end{array}\right) \\
& =\left(\begin{array}{cc}
A^{*} C & \left(B B^{\dagger} A\right)^{*} D \\
\left(A A^{\dagger} B\right)^{*} C & B^{*} D
\end{array}\right) \\
& =\left(\begin{array}{c}
A^{*} C A^{*} D \\
B^{*} C B^{*} D
\end{array}\right) \\
& =(A B)^{*}(C D),
\end{aligned}
$$


and

$$
(A B)(A B)^{*}=A A^{*}+B B^{*}=C A^{*}+D B^{*}=(C D)(A B)^{*},
$$

which according to $(1.2)$ show that $(A B) \stackrel{*}{\leq}(C D)$.

For the left-star orderings, we have a similar result.

Theorem 2 Let $A, C \in C^{m \times n}$ and $B, D \in C^{m \times k}$ be star-ordered as $A * \leq C, B * \leq D$.

If $R(A)=R(B)$, then $(A B) * \leq(C D)$.

Proof. In view of (1.4), according to the assumptions, we have

$$
(A B)^{*}(A B)=(A B)^{*}(C D) .
$$

On the other hand, on account of (1.4), from the conditions $A_{*} \leq C$ and $B_{*} \leq D$, we have $R(A) \subseteq R(C)$ and $R(B) \subseteq R(D)$, which imply that $R(A B) \subseteq R(C D)$. According to (1.4), we have $(A B) * \leq(C D)$.

Theorem 3 Let $A, C \in C^{m \times n}$ and $B, D \in C^{m \times k}$ be star-ordered as $(A B) \stackrel{*}{\leq}(C D)$. If $A \stackrel{*}{\leq} C($ or $B \stackrel{*}{\leq} D)$, then $B \stackrel{*}{\leq} D($ or $A \stackrel{*}{\leq} C)$. Moreover, the condition $A \stackrel{*}{\leq} C($ or $B \stackrel{*}{\leq} D)$ can be replaced by $A \leq * C$ (or $B \leq * D)$.

Proof. The proof is trivial and therefore omitted.

Since $A \stackrel{*}{\leq} B$ and $A \leq * B$ are equivalent to $A^{*} \stackrel{*}{\leq} B^{*}$ and $A^{*} * \leq B^{*}$, respectively, therefore, for the rowwise partitioned matrix we have the similar results.

Corollary 1 Let $A, C \in C^{m \times n}$ and $B, D \in C^{k \times n}$ be star-ordered as $A \stackrel{*}{\leq} C, B \stackrel{*}{\leq} D$. If $R$ $\left(A^{*}\right)=R\left(B^{*}\right)$, then $\left(\begin{array}{l}A \\ B\end{array}\right) \stackrel{*}{\leq}\left(\begin{array}{l}C \\ D\end{array}\right)$.

Corollary 2 Let $A, C \in C^{m \times n}$ and $B, D \in C^{k \times n}$ be star-ordered as $A \leq * C, B \leq * D$. If $R$ $(A *)=R(B *)$, then $\left(\begin{array}{l}A \\ B\end{array}\right) \leq *\left(\begin{array}{l}C \\ D\end{array}\right)$.

Corollary 3 Let $A, C \in C^{m \times n}$ and $B, D \in C^{k \times n}$ be star-ordered as $\left(\begin{array}{l}A \\ B\end{array}\right) \stackrel{*}{\leq}\left(\begin{array}{c}C \\ D\end{array}\right)$. If $A * \leq C($ or $B * \leq D)$, then $B \stackrel{*}{\leq} D($ or $A \stackrel{*}{\leq} C)$.

Specially, we present the following results without proofs.

Theorem 4 Let $A, B \in C^{m \times n}, C \in C^{m \times k}$ and $D \in C^{k \times n}$. Then

(1) If $A \stackrel{*}{\leq} B^{\text {and }} R(C) \subseteq R(A)$, then $(A C) \stackrel{*}{\leq}(B C)$ and $(C A) \stackrel{*}{\leq}(C B)$. Moreover, both $(A C) \stackrel{*}{\leq}(B C)$ and $(C A) \stackrel{*}{\leq}(C B)$ imply $A \stackrel{*}{\leq} B$, even though $R(C) \nsubseteq R(A)$.

(2) If $A * \leq B$ and $R(C) \subseteq R(A)$, then $(A C) * \leq(B C)$ and $(C A) * \leq(C B)$.

(3) If $A \stackrel{*}{\leq} B^{\text {and }} R\left(D^{*}\right) \subseteq R\left(A^{*}\right)$, then $\left(\begin{array}{l}A \\ D\end{array}\right) \stackrel{*}{\leq}\left(\begin{array}{c}B \\ D\end{array}\right)$ and $\left(\begin{array}{l}D \\ A\end{array}\right) \stackrel{*}{\leq}\left(\begin{array}{l}D \\ B\end{array}\right)$. Moreover, both $\left(\begin{array}{l}A \\ D\end{array}\right) \stackrel{*}{\leq}\left(\begin{array}{c}B \\ D\end{array}\right)$ and $\left(\begin{array}{l}D \\ A\end{array}\right) \stackrel{*}{\leq}\left(\begin{array}{l}D \\ B\end{array}\right)$ imply $A \stackrel{*}{\leq} B$ even though $R(D *) \oplus R\left(A^{*}\right)$.

(4) If $A \leq * B$ and $R\left(D^{*}\right) \subseteq R\left(A^{*}\right)$, then $\left(\begin{array}{l}A \\ D\end{array}\right) \leq *\left(\begin{array}{c}B \\ D\end{array}\right)$ and $\left(\begin{array}{l}D \\ A\end{array}\right) \leq *\left(\begin{array}{l}D \\ B\end{array}\right)$.

Next, we use some examples to illustrate the above results. The case (1) shows that the condition $R(C) \subseteq R(A)$ is sufficient but not necessary. For example, we take the matrices 


$$
A=\left(\begin{array}{ll}
0 & 1 \\
0 & 0
\end{array}\right) \text { and } B=\left(\begin{array}{ll}
0 & 1 \\
1 & 0
\end{array}\right) \text {. }
$$

It is easy to verify that $A \stackrel{*}{\leq} B$. For $C=\left(\begin{array}{l}0 \\ 1\end{array}\right), R(C) \nsubseteq R(A)$, and a simple computation shows that $(A C)^{*}(A C) \neq(A C)^{*}(B C)$. For $C=\left(\begin{array}{l}1 \\ 0\end{array}\right), R(C) \subset R(A)$, and we have $(A C) \stackrel{*}{\leq}(B C)$ as well as $(C A) \stackrel{*}{\leq}(C B)$. On the other hand, we take the matrices

$$
A=\left(\begin{array}{ll}
1 & 0 \\
1 & 0 \\
0 & 0
\end{array}\right), B=\left(\begin{array}{ll}
1 & 0 \\
1 & 0 \\
0 & 1
\end{array}\right) \text { and } C=\left(\begin{array}{l}
1 \\
0 \\
0
\end{array}\right) \text {. }
$$

We can verify that $(A C) \stackrel{*}{\leq}(B C)$. Although $R(C) \oplus R(A)$, we have $A \stackrel{*}{\leq} B$.

Mitra [11] pointed out that the star ordering has the property that if $C{ }^{*} A_{A}$ and $C \stackrel{*}{\leq} B$, then $2 C \stackrel{*}{\leq} A+B$. Moreover, it is well known that the Löwner ordering has the property that for Hermitian nonnegative definite matrices $A, B, C$ and $D$, if $A \leq_{L} C$ and $B \leq_{L} D$, then $A+B \leq_{L} C+D$. A direct consideration is to see whether the star ordering has the same property. And the solution is given in the following.

Theorem 5 Let $A, B, C, D \in C^{m \times n}$, and $A^{*} \leq C, B \stackrel{*}{\leq}$. If $R(A)=R(B)$ and $R\left(A^{*}\right)=R$ $\left(B^{*}\right)$, then $A+B \stackrel{*}{\leq} C+D$.

Proof. The proof is trivial and therefore omitted.

\section{Minus partial ordering}

In this section, we present some results on the minus orderings of the matrix product and block matrices. In our development, we will use the following preliminary results for our further discussion.

Lemma 1 [12] Let $A \in C^{m \times n}, B \in C^{n \times k}$. Then

$$
r(A B)=r(B)-\operatorname{dim}(R(B) \cap N(A)) .
$$

Baksalary et al. [13] established a formula for the Moore-Penrose inverse of a columnwise partitioned matrix. Here, we state it as given below.

Lemma 2 Let $A \in C^{m \times n}$ and be partioned as $A=\left(A_{1} A_{2}\right)$. Then the following statements are equivalent:

(1) $A^{\dagger}=\left(\begin{array}{l}A_{1}^{\dagger}-A_{1}^{\dagger} A_{2}\left(Q_{1} A_{2}\right)^{\dagger} \\ A_{2}^{\dagger}-A_{2}^{\dagger} A_{1}\left(Q_{2} A_{1}\right)^{\dagger}\end{array}\right)$,

(2) $R\left(A_{1}\right) \cap R\left(A_{2}\right)=\{0\}$,

where $Q_{i}=I_{m}-A_{i} A_{i}^{\dagger}, i=1,2$.

Lemma 3 [14] Let $A \in C^{m \times n}, B \in C^{m \times k}$, such that $R(B) \subseteq R(A)$. Then

$$
(A B)^{\dagger}=\left(\begin{array}{c}
A^{\dagger}-A^{\dagger} B M^{-1} B^{*}\left(A^{\dagger}\right)^{*} A^{\dagger} \\
M^{-1} B^{*}\left(A^{\dagger}\right)^{*} A^{\dagger}
\end{array}\right)
$$

where $M=I+B^{*}\left(A^{\dagger}\right)^{*} A^{\dagger} B$.

It is easy to verify that, for a full column rank matrix $C$ with proper size, the minus orders $A \leq B$ and $C A \leq C B$ are equivalent, but if $C$ is not a full column rank matrix, this 
implication may be not true. The following theorem shows that when the implication is true.

Theorem 6 Let $A, B \in C^{m \times n}, C \in C^{k \times m}$. Then any two of the following statements imply the third:

(1) $A \bar{\perp} B$,

(2) $C A \overline{\leq} C B$,

(3) $\operatorname{dim}(R(B-A) \cap N(C))=\operatorname{dim}(R(B) \cap N(C))-\operatorname{dim}(R(A) \cap N(C))$.

Proof. Applying Lemma 1, we have

$$
\begin{aligned}
r(C B-C A)= & r(C(B-A))=r(B-A)-\operatorname{dim}(R(B-A) \cap N(C)), \\
& r(C B)=r(B)-\operatorname{dim}(R(B) \cap N(C)), \\
& r(C A)=r(A)-\operatorname{dim}(R(A) \cap N(C)) .
\end{aligned}
$$

Hence,

$$
\begin{aligned}
& (r(B-A)-r(B)+r(A))-(r(C B-C A)-r(C B)+r(C A)) \\
& \quad=\operatorname{dim}(R(B-A) \cap N(C))+\operatorname{dim}(R(A) \cap N(C))-\operatorname{dim}(R(B) \cap N(C)) .
\end{aligned}
$$

On account of (1.6) this theorem can be easily obtained.

Similarly, we can prove the following results.

Corollary 4 Let $A, B \in C^{m \times n}, C \in C^{n \times k}$. Then any two of the following statements imply the third:

(1) $A \overline{\leq} B$,

(2) $A C \dot{\leq} B C$,

(3) $\operatorname{dim}\left(R\left(B^{*}-A^{*}\right) \cap N\left(C^{*}\right)\right)=\operatorname{dim}\left(R\left(B^{*}\right) \cap N\left(C^{*}\right)\right)-\operatorname{dim}\left(R\left(A^{*}\right) \cap N\left(C^{*}\right)\right)$.

Summarizing Theorem 6, Corollary 4 and $N(C)=R^{\perp}\left(C^{*}\right)$, the following results are obtained immediately.

Corollary 5 Let $A, B \in C^{m \times n}$. Then the following statements are equivalent:

(1) $A \overline{\leq} B$,

(2) $B^{\dagger} A \bar{\perp} B^{\dagger}$ Band $R(A) \subseteq R(B)$,

(3) $A B^{\dagger} \leq B B^{\dagger}$ and $R\left(A^{*}\right) \subseteq R\left(B^{*}\right)$.

Furthermore,

$$
\begin{aligned}
& A B^{\dagger} \leq B B^{\dagger} \text { and } R(A) \subseteq R(B) \Leftrightarrow B^{\dagger} A B^{\dagger} \leq B^{\dagger} \text { and } R(A) \subseteq R(B) \\
& B^{\dagger} A \overline{\leq} B^{\dagger} B \text { and } R\left(A^{*}\right) \subseteq R\left(B^{*}\right) \Leftrightarrow B^{\dagger} A B^{\dagger} \leq B^{\dagger} \text { and } R\left(A^{*}\right) \subseteq R\left(B^{*}\right)
\end{aligned}
$$

and

$$
A \subseteq B \Leftrightarrow B^{\dagger} A B^{\dagger} \leq B^{\dagger}, R(A) \subseteq R(B) \text { and } R\left(A^{*}\right) \subseteq R\left(B^{*}\right) .
$$

In the previous section, we study the star ordering of block matrix. A similar consequence on the minus ordering is established as below.

Theorem 7 Let $A, C \in C^{m \times n}$, and $B, D \in C^{m \times k}$ be minus ordered as $A \overline{\leq} C, B \leq D$. If $R$ (C) $\cap R(D)=\{0\}$, then $(A B) \sum(C D)$.

Proof. From $A \leq C$ and $B \leq D$, in view of (1.7), it follows that

$$
A C^{\dagger} C=A, C C^{\dagger} A=A(\text { or } R(A) \subseteq R(C)), A C^{\dagger} A=A ;
$$


and

$$
B D^{\dagger} D=B, D D^{\dagger} B=B(\text { or } R(B) \subseteq R(D)), B D^{\dagger} B=B ;
$$

The conditions of the middle part of (3.1) and (3.2) show that

$$
R(A B) \subseteq R(C D) \text { or }(C D)(C D)^{\dagger}(A B)=(A B)
$$

According to Lemma 2 and the assumption $R(C) \cap R(D)=\{0\}$, we have

$$
(C D)^{\dagger}=\left(\begin{array}{c}
C^{\dagger}-C^{\dagger} D\left(Q_{C} D\right)^{\dagger} \\
D^{\dagger}-D^{\dagger} C\left(Q_{D} C\right)^{\dagger}
\end{array}\right)
$$

where $Q_{C}=I_{m}-C C^{\dagger}$ and $Q D=I_{m}-D D^{\dagger}$.

From (3.1) and (3.2), we can verify the following equalities

$$
\begin{aligned}
& (A B)(C D)^{\dagger}(C D)=(A B), \\
& (A B)(C D)^{\dagger}(A B)=(A B) .
\end{aligned}
$$

On account of (1.7), combining (3.3), (3.4) and (3.5) shows that $(A B) \leq(C D)$ Note that, $A \leq C$ and $B \leq D$ lead to $R(A) \subseteq R(C)$ and $R(B) \subseteq R(D)$, hence, the condition $R(C) \cap R(D)=\{0\}$ implies that $R(A) \cap R(B)=\{0\}$. Therefore, this theorem can also be proved by Definition (1.6).

Since

$$
\begin{aligned}
r[(C D)-(A B)] & =r(C-A D-B) \\
& =r(C-A)+r(D-B) \\
& =r(C)+r(D)-r(A)-r(B) \\
& =r(C D)-r(A B),
\end{aligned}
$$

hence, $(A B) \bar{\Sigma}(C D)$.

The following statement can be deduced from Lemma 3.

Theorem 8 Let $A, C \in C^{m \times n}$ be minus ordered as $A \overline{\leq} C$, and $B, D \in C^{m \times k}$. If $R(D) \subseteq$ $R(C)$, then $(A B) \bar{\Sigma}(C D)$ if and only if $B=A C^{\dagger} D$.

Corollary 6 Let $A, C \in C^{m \times n}$ be minus ordered as, $A \leq C$, and $B, D \in C^{k \times n}$.

(1) If $B \overline{\leq}$ Dand $R\left(C^{*}\right) \cap R\left(D^{*}\right)=\{0\}$, then $\left(\begin{array}{l}A \\ B\end{array}\right) \bar{\Sigma}\left(\begin{array}{l}C \\ D\end{array}\right)$.

(2) If $R\left(D^{*}\right) \subseteq R\left(C^{*}\right)$, then $\left(\begin{array}{l}A \\ B\end{array}\right) £\left(\begin{array}{l}C \\ D\end{array}\right)$ if and only if $B=D C^{\dagger} A$.

\section{Acknowledgements}

This work is supported by Natural Science Foundation Project of CQ CSTC(Grant No. 2010BB9215). The authors would like to thank the anonymous referees for constructive comments that improved the contents and presentation of this paper.

Authors' contributions

$\mathrm{XL}$ carried out the main part of this article. All authors read and approved the final manuscript.

\section{Competing interests}

The authors declare that they have no competing interests.

Received: 20 February 2011 Accepted: 13 September 2011 Published: 13 September 2011 


\section{References}

1. Ben-Israel, A, Greville, TNE: Generalized Inverses: Theory and Applications. Springer, New York, 2 (2003)

2. Drazin, MP: Natural structures on semigroups with involution. Bull Am Math Soc. 84, 139-141 (1978). doi:10.1090/500029904-1978-14442-5

3. Baksalary, JK, Mitra, SK: Left-star and right-star partial orderings. Linear Algebra Appl. 149, $73-89$ (1991). doi:10.1016/ 0024-3795(91)90326-R

4. Hartwig, RE: How to partially order regular elements. Math Jpn. 25, 1-13 (1980)

5. Nambooripad, KSS: The natural partial order on a regular semigroup. Proc Edinb Math Soc. 23, 249-260 (1980). doi:10.1017/S0013091500003801

6. Hartwig, RE, Styan, GPH: On some characterizations of the "star" partial ordering for matrices and rank subtractivity. Linear Algebra Appl. 82, 145-161 (1986). doi:10.1016/0024-3795(86)90148-5

7. Baksalary, JK, Hauke, J, Liu, X, Liu, S: Relationships between partial orders of matrices and their powers. Linear Algebra Appl. 379, 277-287 (2004)

8. Baksalary, JK, Baksalary, OM, Liu, X: Further properties of the star, left-star, right-star, and minus partial orderings. Linear Algebra Appl. 375, 83-94 (2003)

9. Baksalary, JK, Baksalary, OM, Liu, X: Further relationships between certain partial orders of matrices and their squares, Linear Algebra Appl. 375, 171-180 (2003)

10. Benitez, J, Liu, X, Zhong, J: Some results on matrix partial orderings and reverse order law. Electron J Linear Algebra. 20, $254-273(2010)$

11. Mitra, SK: Infimum of a pair of matrices. Linear Algebra Appl. 105, 163-182 (1988). doi:10.1016/0024-3795(88)90010-9

12. Marsaglia, G, Styan, GPH: Equalities and inequalities for ranks of matrices. Linear Multilinear Algebra. 2, 269-292 (1974). doi:10.1080/03081087408817070

13. Baksalary, JK, Baksalary, OM: Particular formulae for the Moore-Penrose inverse of a columnwise partitioned matrix. Linear Algebra Appl. 421, 16-23 (2007). doi:10.1016/j.laa.2006.03.031

14. Wang, S, Yang, Z: Generalized inverse for matrices and its applications. Beijing University of Technology Press, Beijing (1996)

doi:10.1186/1029-242X-2011-54

Cite this article as: Liu and Yang: Some results on the partial orderings of block matrices. Journal of Inequalities and Applications 2011 2011:54.

\section{Submit your manuscript to a SpringerOpen ${ }^{\circ}$} journal and benefit from:

- Convenient online submission

- Rigorous peer review

- Immediate publication on acceptance

- Open access: articles freely available online

- High visibility within the field

- Retaining the copyright to your article

Submit your next manuscript at $\gg$ springeropen.com 